\title{
Maruhn-Greiner Maximum of Uranium Fission for Confirmation of Low Energy Nuclear Reactions LENR via a Compound Nucleus with Double Magic Numbers
}

\author{
H. Hora · G. H. Miley
}

Published online: 26 July 2008

(C) Springer Science+Business Media, LLC 2008

\section{Erratum to: J Fusion Energ (2007) 26(4):349-355 DOI 10.1007/s10894-007-9080-7}

The third paragraph after Eq. 8 in the original paper has to be clarified with regard to the numbers about the generation of the deuteron clusters and their properties for BoseEinstein condensation (BEC).

The deuterium ions are space charge neutral until a diameter of 2 to $3 \mathrm{pm}$ due to the screening factor 13 which is 2.6 times higher than accepted in high temperature plasmas. For smaller distances their positive charge will cause repulsion. Only for this small distance the mutual force possibly as a Casimir effect results in an energy density to attach the screened deuterons to clusters with 2 to $3 \mathrm{pm}$ deuteron distance such that their thermal energy at room temperature is not sufficient to escape the cluster. Their spin 1 as bosons permits a state of Bose-Einstein condensation because their de Brolie wavelength is $1.2 \AA$ while at $100 \%$ loading in palladium their initial distance is $2.5 \AA$. The quantum state does not provide a defined location within the cluster such that the whole cluster of 156 deuterons for the nuclear compound reaction Eq. 10 occurs at a long distance $(2 \mathrm{pm})$ with a Pd nucleus. This reaction was observed fully reproducibly after 3 weeks interaction [3-5] and therefore is basically different from deuterons in the Maxwellian gas within the metal for statistically very rare generated clusters with 10 times higher density where the BEC permits DD reactions according to the reactions [1] which therefore are stochastic and basically different from LENR [6].

The online version of the original article can be found under doi: 10.1007/s10894-007-9080-7.

\footnotetext{
H. Hora $(\bowtie)$

Department of Theoretical Physics, University of New South

Wales, Sydney 2052, Australia

e-mail: h.hora@unsw.edu.au

G. H. Miley

Department of Nuclear, Plasma and Radiological Engineering,

University of Illinois, Urbana, IL 61801, USA
} 